\title{
Forecasting tourist arrivals using time-varying parameter structural time series models
}

\author{
HAIYAN SONG $\dagger^{1}$, GANG LI*, STEPHEN F. WITT* AND GEORGE \\ ATHANASOPOULOS
}

$\uparrow$ School of Hotel and Tourism Management

The Hong Kong Polytechnic University

Hung Hom, Kowloon

Hong Kong SAR

*Faculty of Management and Law

University of Surrey

Guildford GU2 7XH

United Kingdom

$\ddagger$ Department of Econometrics and Business Statistics

Monash University

VIC 3800

Australia

\begin{abstract}
Empirical evidence has shown that seasonal patterns of tourism demand and the effects of various influencing factors on this demand tend to change over time. To forecast future tourism demand accurately requires appropriate modelling of these changes. Based on the structural time series model (STSM) and the time-varying parameter (TVP) regression approach, this study develops the causal STSM further by introducing TVP estimation of the explanatory variable coefficients, and therefore combines the merits of the STSM and TVP model. This new model, the TVP-STSM, is employed for modelling and forecasting quarterly tourist arrivals to Hong Kong from four key source markets: China, South Korea, the UK and the USA. The empirical results show that the TVP-STSM outperforms all seven competitors, including the basic and causal STSMs and the TVP model for oneto four-quarters-ahead ex post forecasts and one-quarter-ahead ex ante forecasts.
\end{abstract}

Keywords: Tourism demand forecasting, Seasonality, Stochastic, State space models

\footnotetext{
${ }^{1}$ Corresponding author (hmsong@ polyu.edu.hk).

This article should be cited as:

Song H, Li G, Witt SF, Athanasopoulos G. (2011) 'Forecasting Tourist Arrivals Using Time-Varying

Parameter Structural Time Series Models'. International Journal of Forecasting, 27 (3), pp. 855-869
} 


\section{Introduction}

Tourism contributes significantly to the economic growth of many countries and regions. Considering the rapid increase in international tourism demand over the last few decades, accurate predictions of future trends of tourism demand are of particular importance to both tourism policymakers and tourism business practitioners. Moreover, in most destinations tourism demand displays significant seasonal variations. Seasonality affects tourism in various different ways and is responsible for difficulties in gaining access to capital, high risks of investment and business failures, the ineffective utilisation of resources and facilities, and difficulties in maintaining a consistent service quality. However, seasonality is not always detrimental to the industry, as the off-peak season has benefits such as time for environmental reclamation and "resident recovery" (Butler, 1994). Regardless of the advantages and disadvantages of seasonality, a comprehensive knowledge of seasonal patterns of tourism demand and the accurate prediction of their future values will contribute to effective planning and operations management, such as staffing, resource allocation and capacity management.

Empirical evidence has shown that seasonal patterns of tourism demand and the effects of various influencing factors on demand tend to change over time. Forecasting future tourism demand accurately therefore requires the appropriate modelling of both seasonality and the effects of the explanatory variables. Structural time series models (STSMs), which specify the trend, seasonal and cycle components of a variable as stochastic, and the time-varying parameter (TVP) regression approach, which relaxes the restriction on the constancy of the demand parameters over time, have both been introduced into tourism demand studies and have demonstrated superior forecasting performance compared to deterministic models. The aim of this study is to construct a new econometric model that develops the causal STSM further by introducing TVP estimation of the explanatory variable coefficients, and therefore combines the merits of the STSM and TVP model. This new TVP-STSM is expected to forecast seasonal tourism demand more accurately than previously used methods. The empirical study evaluates the forecasting accuracy of the proposed model for forecasting tourist arrivals to Hong Kong from four key source markets: China, South Korea, the UK and the USA.

\section{Modelling and forecasting seasonal tourism demand}

A large body of literature on seasonal tourism demand analysis and forecasting has been published over the last two decades, and has contributed significantly to our understanding of the features of seasonal tourism demand. However, there are potential problems with these studies. Earlier studies tended to regard the patterns of seasonality in tourism demand as constant. However, due to various changes, such as climate and weather conditions, the popularity of tourist activities and destinations, technology and politics, seasonality is not deterministic. To overcome the assumption of deterministic seasonality, the STSM, initially proposed by Harrison and Stevens (1976) and later refined by Harvey (1989), was introduced into seasonal tourism demand studies. 
The basic STSM without the inclusion of explanatory variables (also known as the basic structural model or BSM) decomposes a time series into its trend, seasonal, cycle and irregular components and regards these components as stochastic. Hence, this model reflects the seasonal variation in tourism demand better than the traditional constant seasonal time series models. However, the BSM (and univariate time series models in general) does not account for the effects of economic determinants on the variable of interest. To overcome this limitation, the BSM was developed further to include causal variables in the model specification (known as the causal structural model or CSM). Both the BSM and the CSM have been applied in the tourism demand forecasting context, and several studies have demonstrated their superior forecasting performance relative to other time series alternatives (see for example González \& Moral, 1995, 1996; Kulendran \& King, 1997; Kulendran \& Witt, 2001; Turner \& Witt, 2001). However, in the studies of both Turner and Witt (2001) and Kulendran and Witt (2003), the CSM produced less accurate forecasts than the BSM.

One possible reason why the CSM could not generate more accurate forecasts than the BSM is related to its treatment of the explanatory variables. Although the seasonality, trend and cycle in the CSM are all regarded as stochastic, the parameters of the explanatory variables are treated as being constant over time. This implies that the economic structure generating the data does not change. In a demand model specified in double-log form, constant parameters suggest that the elasticities of tourism demand are constant over the sample period, which is very restrictive and often unrealistic. In reality, the changing economic environment may induce people to react differently to a given stimulus at different points in time. As the modifications to the environment are transitory or ambiguous in some situations, the changes in the coefficients are likely to follow a stochastic process (Lucas, 1976). However, the CSM does not take this into account, which may contribute to its unsatisfactory forecasting performance.

To overcome the above limitations of the traditional fixed-parameter estimation of demand models, the time-varying parameter (TVP) modelling approach was introduced into the tourism context in the late 1990s. This approach relaxes the restriction on the constancy of the coefficients of the explanatory variables and allows for stochastic parameters so that it can better reflect the evolution of demand elasticities over time. Previous empirical studies employing the TVP technique have shown that models that incorporate the TVP approach tend to generate more accurate forecasts than other econometric models, especially in the short term. For example, Song and Witt (2000), Song, Witt, and Jensen (2003) and Witt, Song, and Louvieris (2003) examined the TVP model's performance in forecasting international tourism demand relative to other fixedparameter econometric models and time series models. The first two studies assess the forecasting accuracy in terms of the error magnitude using the mean absolute percentage error (MAPE) and root mean square percentage error (RMSPE) measures. In both studies, the TVP model outperforms all of the competitors, including the autoregressive distributed lag model (ADLM), the vector autoregressive (VAR) model, ECMs and the naïve no-change model in the one-year-ahead forecasting comparison. Witt et al. (2003) investigate the forecasting performance of the TVP model in terms of both error 
magnitude and directional change. The comparison results show that the TVP model is ranked second best amongst seven candidates in a one year ahead forecasting competition in both assessments. All three empirical studies show that the TVP technique is ranked either first or second for short-run (specifically one year ahead) forecasting, which implies that the TVP model is highly suitable for short-run tourism planning purposes. However, all of the above applications of the TVP model use annual tourism demand data and the influence of seasonality on tourism demand is not examined in these studies. The above TVP models can readily incorporate seasonal dummies in order to forecast seasonal tourism demand (see, for example, Shen, Li, \& Song, 2008), but this implies deterministic seasonality.

The current study represents the first attempt to incorporate time-varying parameters in structural time series models for seasonal tourism demand forecasting. This new modelling approach provides a comprehensive analysis of seasonal demand and is expected to improve forecasting accuracy. The TVP-STSM is applied to forecasting the quarterly demand for Hong Kong tourism by tourists from four key source markets. As with most tourist destinations, Hong Kong tourism demand experiences significant seasonality. The forecasting accuracy of the newly developed TVP-STSM is compared with that of commonly used time series and econometric forecasting models. The empirical results of this study will provide very useful information for the key tourism players and public agencies in formulating their tourism policies and evaluating the effectiveness of these.

\section{Methodology}

The TVP-STSM developed here is based on the TVP model and the STSM, which have common technical features such as being written in state space form and being estimated using the Kalman filter algorithm. Based on the advantages of the two models and their common technical foundation, the TVP-STSM is expected to show improved forecasting ability when dealing with seasonal data.

\subsection{TVP-STSM specification}

The TVP-STSM can be represented in the following state space form (SSF):

$$
\begin{aligned}
& y_{t}=\mu_{t}+\psi_{t}+\gamma_{t}+X_{t} \Gamma_{t}+\varepsilon_{t} \quad \varepsilon_{t} \sim \operatorname{NID}\left(0, H_{t}\right) \\
& \mu_{t+1}=\mu_{t}+\beta_{t}+v_{t} \quad v_{t} \sim \operatorname{NID}\left(0, \sigma_{v}^{2}\right) \\
& \beta_{t+1}=\beta_{t}+\delta_{t} \quad \delta_{t} \sim \operatorname{NID}\left(0, \sigma_{\delta}^{2}\right) \\
& {\left[\begin{array}{l}
\psi_{t+1} \\
\psi_{t+1}^{*}
\end{array}\right]=\rho\left[\begin{array}{cc}
\cos \tau & \sin \tau \\
-\sin \tau & \cos \tau
\end{array}\right]\left[\begin{array}{l}
\psi_{t} \\
\psi_{t}^{*}
\end{array}\right]+\left[\begin{array}{c}
\omega_{t} \\
\omega_{t}^{*}
\end{array}\right] \quad \omega_{t}, \omega_{t}^{*} \sim \operatorname{NID}\left(0, \sigma_{\omega}^{2}\right)}
\end{aligned}
$$


$\gamma_{t+1}=\sum_{j=1}^{s-1} \gamma_{t+1-j}+\kappa_{t} \quad \kappa_{t} \sim N I D\left(0, \sigma_{\kappa}^{2}\right)$

$\Gamma_{t+1}=T_{t} \Gamma_{t}+R_{t} \eta_{t}, \quad \Gamma_{1} \sim N\left(K_{1}, P_{1}\right), \quad \eta_{t} \sim N I D\left(0, Q_{t}\right)$,

where $y_{t}$ is a univariate time series, decomposed into its unobservable components, including a trend component $\left(\mu_{t}\right)$, a cycle component $\left(\psi_{t}\right)$, a seasonal component $\left(\gamma_{t}\right)$ and an irregular component $\left(\varepsilon_{t}\right) . X_{t}$ is a vector of causal variables and $\Gamma_{t}$ is the corresponding vector of coefficients. As equation (1) suggests, the TVP-STSM is linear in state variables $\left(\mu_{t}, \psi_{t}, \gamma_{t}\right.$ and $\left.\Gamma_{t}\right)$, which are governed in turn by first-order linear transition equations (equations (2) to (6)). Equations (2) and (3) specify the stochastic trend, where $\beta_{t}$ is the slope of the trend. Equation (4) refers to a stochastic cycle specification, where $\tau \in[0, \pi]$ is the cyclical frequency, $\rho \in[0,1]$ is the damping factor of the cycle, and $\psi_{t}^{*}$ appears by construction. The seasonal component is defined in equation (5) in such a stochastic way that the seasonal pattern is allowed to change over time, where $s$ is the number of seasons per year. It is often preferable to express the stochastic seasonality in trigonometric form, similarly to the specification of the cyclical component (see Harvey, 1989, for more details). The white noise disturbances of the trend, cycle and seasonal equations (equations (2) to (5)) are mutually independent and $\sigma_{v}^{2}, \sigma_{\delta}^{2}, \sigma_{\omega}^{2}$ and $\sigma_{\kappa}^{2}$ are the corresponding variances. Unlike traditional regression models, the coefficients of the causal variables in a TVP-STSM are specified as timevarying, as equation (6) suggests. In most economic applications, it is assumed that $T_{t}=I$, where $I$ is the identity matrix. In this case, $\Gamma_{t}$ follows a multivariate random walk, and $H_{t}, R_{t}, Q_{t}$ and $T_{t}$ are system matrices that are initially assumed to be known.

With regard to the estimation of the TVP-STSM written in SSF, the filtering and smoothing algorithms can be employed, conditional on known state and error system matrices. As for the unknown parameters in these matrices, they are estimated using maximum likelihood estimation methods (Koopman et al., 2007). Detailed explanations and further references with regard to the specification and estimation of state space models are given by Durbin and Koopman (2001), Hamilton (1994a,b), Harvey (1989) and Koopman, Shephard, and Doornik (1999).

\subsection{Specifications of alternative models}

The above TVP-STSM is a more general form of a structural time series model. The BSM, CSM and TVP models can all be regarded as special cases of the TVP-STSM specification.

If no causal variables are incorporated into equation (1), the TVP-STSM is reduced to a BSM: $y_{t}=\mu_{t}+\psi_{t}+\gamma_{t}+\varepsilon_{t}$. If causal variables are included but their parameters are estimated as time-invariant, the TVP-STSM is reduced to a CSM: $y_{t}=\mu_{t}+\psi_{t}+\gamma_{t}+X_{t} \Gamma+\varepsilon_{t}$, where $\Gamma$ does not have a time subscript. If no unobservable 
components (apart from $\varepsilon_{t}$ ) are included in the model, the TVP-STSM is reduced to the TVP model: $y_{t}=X_{t} \Gamma_{t}+\varepsilon_{t}$. Where seasonal data are used, deterministic seasonal dummies can readily be added to the TVP model. However, such a model is unable to analyse the stochastic seasonality or cycles of a time series.

With a more general specification, the TVP-STSM embodies the various advantages of the CSM and TVP model. This new model is better able to explain the dynamics of an economic series, and is expected to further enhance forecasting performance. The merits of the TVP-STSM are empirically demonstrated in the following section.

\section{Empirical results and discussion}

The empirical application focuses on modelling and forecasting tourist arrivals to Hong Kong from four representative leading source markets, namely China, South Korea, the UK and the USA. The dynamic effects of the seasonality, trend, cycle and various influencing factors on the demand for tourism in Hong Kong are estimated for these four key source markets. In addition, the forecasting accuracy of the TVP-STSM is compared with that of other time series and econometric models which are commonly used in tourism demand studies.

\subsection{The data and variables}

The following demand function is proposed for modelling the demand for Hong Kong tourism by residents from a particular origin country:

$$
\ln D_{i}=f\left(\ln Y_{i}, \ln P_{h k i}, \ln P_{s i}, \text { Dummies, Trend, Seasonal, Cycle }\right),
$$

where "ln" stands for the natural logarithm; $D_{i}$ is tourism demand, for which the variable of tourist arrivals from origin country $i$ is used as a proxy; $Y_{i}$ is the income level in origin country $i$, measured by the gross domestic product (GDP) at constant prices; and $P_{h k i}$ is the tourism price in Hong Kong relative to that of the origin country $i$, adjusted by the relevant exchange rates, i.e.,

$$
P_{h k i}=\frac{\left(C P I_{h k} / E X_{h k}\right)}{\left(C P I_{i} / E X_{i}\right)}
$$

where $C P I_{h k}$ and $C P I_{i}$ are the $C P I s$ for Hong Kong and origin country $i$ respectively; and $E X_{h k}$ and $E X_{i}$ are the exchange rates between the Hong Kong dollar and the US dollar, and between the currency of origin country $i$ and the US dollar, respectively. Ideally, the tourism price should include tourists' living costs, as well as the travel cost to Hong Kong, but due to the difficulties in obtaining reliable travel cost data, the own price variable only contains the living cost component. Furthermore, it has been reported that the travel cost variable is insignificant in many tourism demand models, such as that of Smeral Witt, and Witt (1992). This is due mainly to the fact that the average economy airfare is not considered to be a good proxy for the travel cost variable. The substitute 
price variable $P_{s i}$ in equation (7) is defined as a weighted average index of selected countries' tourism prices. In the selection of substitute destinations, not only the geographic characteristics but also the cultural characteristics are taken into account. Singapore, Taiwan, Thailand, South Korea and Japan were selected as substitute destinations in this study. In the case where South Korea is the origin country under consideration, it is excluded from the substitute price calculation. The substitute price index is calculated by weighting the exchange-rate adjusted CPI of each substitute destination according to its share of visitor arrivals, and is given as,

$$
P_{s i}=\sum_{j=1}^{5}\left(C P I_{j} / E X_{j}\right) w_{j},
$$

where $w_{j}$ is the market share of substitute destination $j$, which is calculated from,

$$
w_{j}=\left[T T A_{j} / \sum_{j=1}^{5} T T A_{j}\right],
$$

where $T T A_{j}$ is the total number of visitor arrivals to country/region $j$. The above explanatory variables are commonly considered in tourism demand studies and have generally been shown to have significant effects on tourism demand (Witt \& Witt, 1995).

In addition to the above explanatory variables, some dummies are included to capture the effects on Hong Kong tourism demand of various one-off events (such as the Gulf war in 1990, the Asian financial crisis in 1997-1998, the handover of Hong Kong to China in 1997, the 9/11 terrorist attack in 2001 and SARS in 2003). The dummy variables take the value of 1 for the period when the one-off event occurs and zero otherwise. In particular, the handover of Hong Kong to China appeared to have had only a short-term effect, and hence the dummy variable is specified as an outlier instead of a step intervention. It should be noted that since a one-off event may have different influences (in either scale or time period) on tourism demand from different source markets, the specification of a given dummy variable may vary across the different models. The trend, seasonal and cycle components are specified as outlined in Section 3, following Koopman, Harvey, Doornik, and Shephard (2007).

In this study, quarterly data are collected over the period 1985Q1-2008Q4, within which the data from 1985Q1 to 2004Q4 are employed for model estimation and the rest are used for forecasting comparisons. Visitor arrivals are initially collected from the statistics of the Hong Kong Tourism Board (HKTB) on a monthly basis, and are then aggregated to give quarterly data. The income, price and exchange rate data are obtained from the International Financial Statistics Yearbooks published by the International Monetary Fund (IMF). 


\subsection{Model estimation}

The general-to-specific approach is adopted for the TVP-STSM estimation. The initial specification of the measurement equation of the TVP-STSM includes trend (level and slope), cycle, seasonal and irregular components, three explanatory variables, and some dummy variables as discussed above. The coefficients of the explanatory variables are specified as time-varying and are modelled as random walk processes. If the variances of the estimated parameters are zero, the coefficients of the explanatory variables are then taken as fixed parameters in the re-estimated model.

With regard to the unobserved components, including the trend, seasonal, cycle and irregular components, it is useful to run BSMs to examine the properties (i.e. stochastic versus deterministic processes) of these components in the various tourist arrivals series. In particular, such an examination helps in identifying whether any cycles exist in the data, so that spurious cycles can be avoided in the TVP-STSM modelling stage. The cyclical property check is particularly useful when a small data sample is used, as spurious cycles are more likely to occur in structural time series modelling. It should be noted that some components feature (relatively weak) stochastic processes in a BSM, but may become deterministic in a TVP-STSM. In other words, including explanatory variables and modelling their coefficients as random walk processes may contribute to explaining the variation in the dependent variable. Therefore, it is worth checking these properties again in a TVP-STSM. Since previous econometric studies have suggested that tourists' incomes, relative tourism prices and prices in competing destinations are the most important determinants of tourism demand for most destinations ( $\mathrm{Li}$, Song, \& Witt, 2005), and are thus able to explain the level of tourism demand to a great extent, it is both appropriate and effective to start with a smooth local linear trend model; i.e., the disturbance in the level equation is set to zero but the disturbance in the slope equation is non-zero, as specified by Koopman et al. (2007, p. 172). Therefore, in the initial TVPSTSM, the trend, seasonal and irregular components are specified as stochastic, and the cycle component is only included if strong evidence is shown in a BSM. Again, the variances of the estimated parameters of these unobserved components will suggest whether or not it is more appropriate to specify them as deterministic. The model is then re-estimated with all components set appropriately. In addition to the pre-selected dummy variables, some country-specific dummies are included, as suggested by the data plots. The computer programme STAMP 8.10 is used to obtain maximum likelihood estimates of the model parameters. STAMP can convert a linear dynamic model such as the proposed TVP-STSM into state space form (a first-order dynamic linear model), and applies the Kalman filter in its maximum likelihood procedure.

Following the above procedure, the general TVP-STSM will be reduced to a simpler specification step by step. The final specification should pass all diagnostic tests, including the residual serial correlation, heteroscedasticity and normality tests. In addition, the estimation results need to meet certain convergence criteria. Since the estimation of a TVP-STSM is a maximisation process that is terminated when three convergence criteria hold (depending on different settings of the critical values), a good TVP-STSM specification should meet high levels of the criteria. In STAMP's estimation 
report, the message of "strong convergence" or "very strong convergence" is expected to be obtained. Once the model passes all of the diagnostic statistics and at least strong convergence is achieved, it will be used for forecasting purposes. Table 1 summarises the results for the four estimated models in the initial estimation sample 1985Q1 to 2004Q4. Figures 1 and 2 illustrate the evolution over time of various estimated parameters in the four models.

Table 1. TVP-STSM estimates and diagnostics for tourist arrivals to Hong Kong.

\begin{tabular}{|c|c|c|c|c|}
\hline & China & Korea & UK & US \\
\hline \multicolumn{5}{|c|}{$\begin{array}{l}\text { Hyperparameters } \\
\text { (Average percentage deviations) }\end{array}$} \\
\hline Level & 0.000 & 0.000 & 0.000 & 0.000 \\
\hline Slope & 0.000 & 0.047 & 0.028 & 0.000 \\
\hline Seasonal & 0.060 & 0.366 & 0.346 & 0.385 \\
\hline Irregular & 0.000 & 1.891 & 1.105 & 0.000 \\
\hline $\ln Y_{i}$ & 1.851 & 1.886 & 1.272 & 1.467 \\
\hline $\ln \mathrm{P}_{h k i}$ & 2.957 & 12.649 & 0.019 & 0.838 \\
\hline $\ln \mathrm{P}_{s i}$ & 0.000 & 0.000 & 0.000 & 0.698 \\
\hline \multicolumn{5}{|l|}{ Coefficients } \\
\hline Level & $\begin{array}{l}5.450 * * * \\
{[0.428]}\end{array}$ & $\begin{array}{l}2.106 \\
{[1.501]}\end{array}$ & $\begin{array}{l}1.697 \\
{[2.871]}\end{array}$ & $\begin{array}{l}4.268 \\
{[3.377]}\end{array}$ \\
\hline Slope & $\begin{array}{l}0.018^{* * *} \\
{[0.003]}\end{array}$ & $\begin{array}{l}0.006 \\
{[0.008]}\end{array}$ & $\begin{array}{l}-0.002 \\
{[0.005]}\end{array}$ & $\begin{array}{l}0.002 \\
{[0.007]}\end{array}$ \\
\hline $\ln Y_{i}$ & $\begin{array}{l}0.483 * * * \\
{[0.1971}\end{array}$ & $\begin{array}{l}1.370 * * \\
{[0.722]}\end{array}$ & $\begin{array}{l}1.614 \\
{[1.401]}\end{array}$ & $\begin{array}{l}0.553 \\
{[1.655]}\end{array}$ \\
\hline $\operatorname{lnP}_{h k i}$ & $\begin{array}{l}-0.166 \\
{[0.299]}\end{array}$ & $\begin{array}{l}-0.772^{*} \\
{[0.584]}\end{array}$ & $\begin{array}{l}-0.106 \\
{[0.160]}\end{array}$ & $\begin{array}{l}-0.587 \\
{[0.613]}\end{array}$ \\
\hline $\operatorname{lnP_{si}}$ & $\begin{array}{l}-0.026 \\
{[0.274]}\end{array}$ & $\begin{array}{l}0.015 \\
{[0.681]}\end{array}$ & $\begin{array}{l}0.781 * * * \\
{[0.309]}\end{array}$ & $\begin{array}{l}-0.037 \\
{[0.331]}\end{array}$ \\
\hline Dum1997 & $\begin{array}{l}0.035 \\
{[0.027]}\end{array}$ & $\begin{array}{l}-0.049 \\
{[0.040]}\end{array}$ & $\begin{array}{l}-0.170^{* * *} \\
{[0.026]}\end{array}$ & $\begin{array}{l}0.040 \\
{[0.025]}\end{array}$ \\
\hline Dum2001 & & & $\begin{array}{l}0.014 \\
{[0.027]}\end{array}$ & $\begin{array}{l}-0.090^{* * * *} \\
{[0.026]}\end{array}$ \\
\hline Dum2003 & $\begin{array}{l}-0.292 * * * \\
{[0.029]}\end{array}$ & $\begin{array}{l}-0.576^{* * *} \\
{[0.041]}\end{array}$ & $\begin{array}{l}-0.626^{* * *} \\
{[0.027]}\end{array}$ & $\begin{array}{l}-0.725^{* * *} \\
{[0.027]}\end{array}$ \\
\hline \multicolumn{5}{|c|}{ Seasonal effects } \\
\hline Seasonal 1 & $\begin{array}{l}0.031^{*} \\
{[0.016]}\end{array}$ & $\begin{array}{l}0.053^{*} \\
{[0.027]}\end{array}$ & $\begin{array}{l}0.037 * * \\
{[0.015]}\end{array}$ & $\begin{array}{l}-0.029 * * \\
{[0.016]}\end{array}$ \\
\hline Seasonal 2 & $\begin{array}{l}-0.006 \\
{[0.007]}\end{array}$ & $\begin{array}{l}-0.023 \\
{[0.018]}\end{array}$ & $\begin{array}{l}-0.033^{* *} \\
{[0.015]}\end{array}$ & $\begin{array}{c}0.013 \\
{[0.015]}\end{array}$ \\
\hline Seasonal 3 & $\begin{array}{l}0.010 \\
{[0.006]}\end{array}$ & $\begin{array}{l}0.007 \\
{[0.017]}\end{array}$ & $\begin{array}{l}-0.069^{* * *} \\
{[0.013]}\end{array}$ & $\begin{array}{l}-0.028^{*} \\
{[0.014]}\end{array}$ \\
\hline Seasonal 4 & $\begin{array}{l}-0.035^{*} \\
{[0.018]}\end{array}$ & $\begin{array}{l}-0.037 \\
{[0.030]}\end{array}$ & $\begin{array}{l}0.065 * * * \\
{[0.014]}\end{array}$ & $\begin{array}{l}0.045^{* * *} \\
{[0.015]}\end{array}$ \\
\hline \multicolumn{5}{|c|}{ Diagnostics } \\
\hline $\begin{array}{l}\text { Normality } \\
\mathrm{H}(23) \\
\mathrm{Q}(10,7) \\
\mathrm{r}(1)\end{array}$ & $\begin{array}{l}2.564 \\
0.775 \\
7.753 \\
-0.079\end{array}$ & $\begin{array}{l}0.030 \\
1.110 \\
6.810 \\
-0.018\end{array}$ & $\begin{array}{l}2.649 \\
2.199 \\
4.728 \\
-0.163\end{array}$ & $\begin{array}{l}5.578 \\
0.594 \\
4.482 \\
-0.024\end{array}$ \\
\hline
\end{tabular}




\begin{tabular}{lllll}
\hline $\mathrm{r}(10)$ & -0.128 & 0.149 & 0.026 & -0.083 \\
p.e.v. & $1.087 \mathrm{e}-3$ & $1.686 \mathrm{e}-2$ & $5.786 \mathrm{e}-4$ & $6.172 \mathrm{e}-4$ \\
$R^{2}$ & 0.995 & 0.865 & 0.971 & 0.960 \\
$R_{s}^{2}$ & 0.746 & 0.178 & 0.955 & 0.962 \\
Convergence & very strong & strong & strong & strong \\
\hline
\end{tabular}

Notes: $*, * *$ and $* * *$ denote significance at $10 \%, 5 \%$ and $1 \%$ levels, respectively (one-tailed tests for the income and own-price variables); the above models are estimated for the initial estimation sample 1985Q1-2004Q4; the values in brackets are standard errors; 'Normality' refers to the corrected Bowman-Shenton error normality statistic, approximately distributed as chi-square (2) under the null hypothesis; $\mathrm{H}(23)$ is a heteroscedasticity statistic distributed as $\mathrm{F}(23,23)$ based on a two-tailed test (Commandeur \& Koopman, 2007); Q(10, 7) is the Box-Ljung statistic distributed as chi-square (7); $r(1)$ and $r(10)$ are the serial correlation coefficients at the equivalent residual lags, approximately normally distributed; p.e.v. refers to the prediction error variance; $R_{s}^{2}$ measures the relative goodness-of-fit against a random walk plus drift and fixed seasonals.
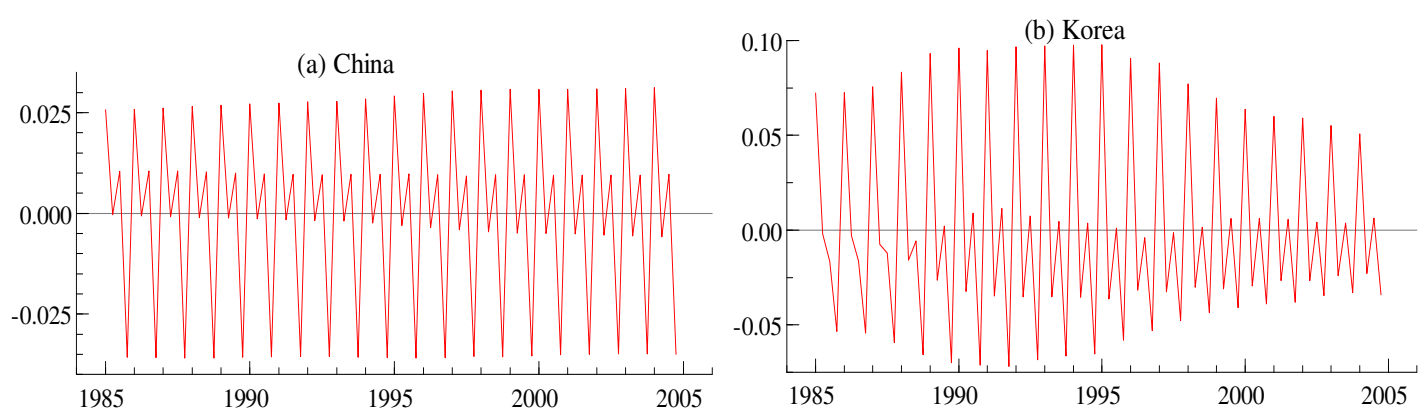

(c) UK
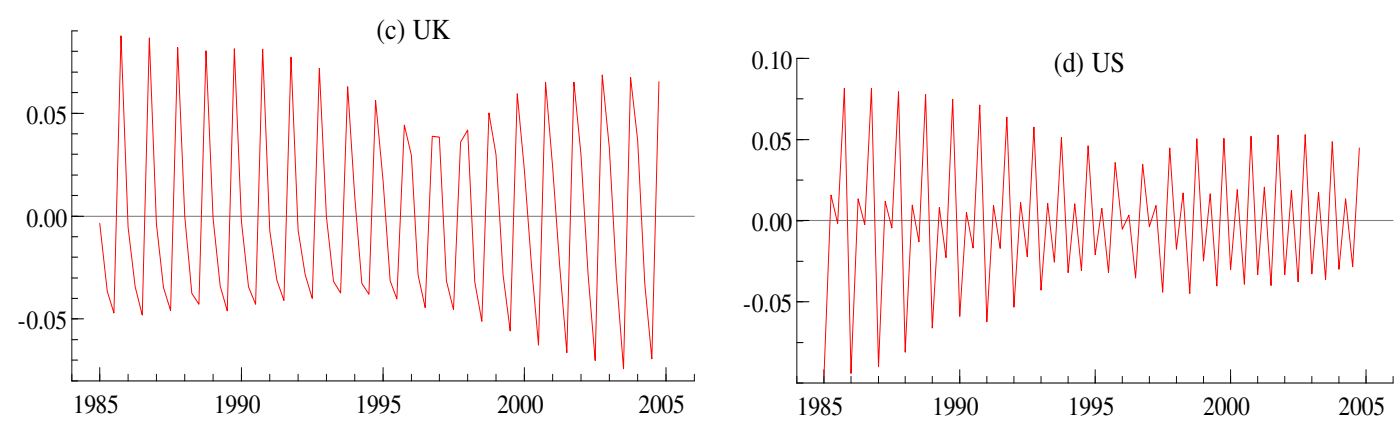

Figure 1. Seasonal components of the estimated TVP-STSMs. 

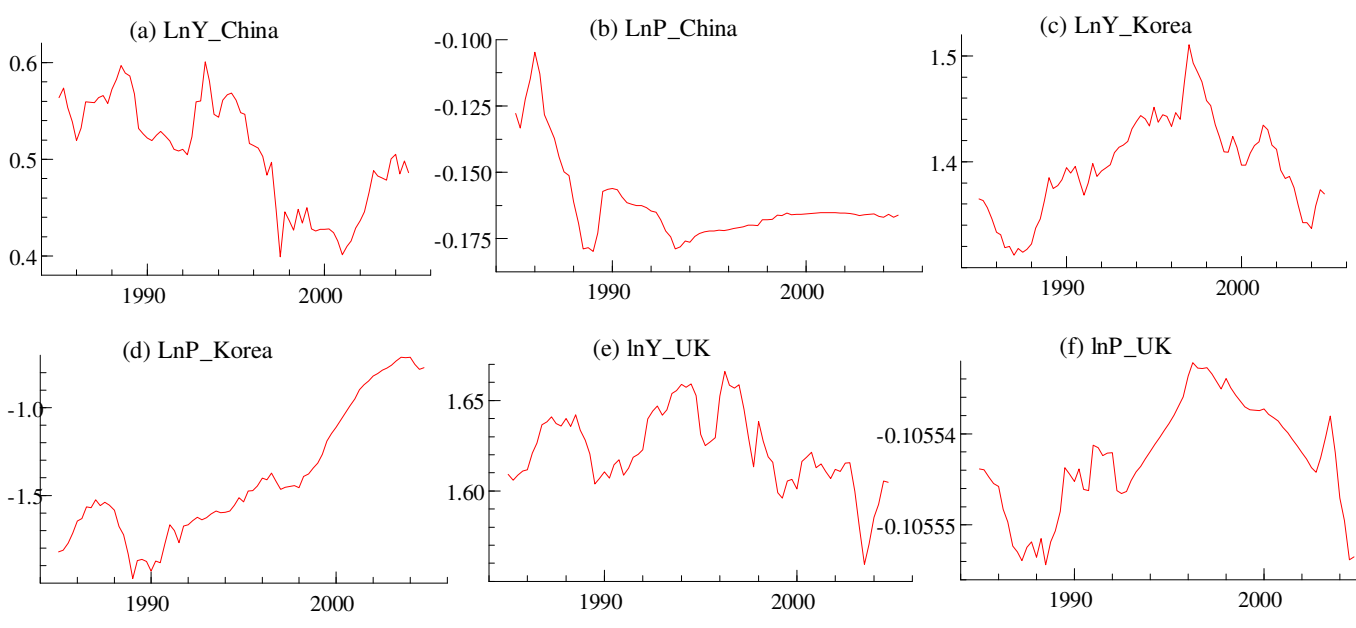

(f) $\ln P_{-} U K$
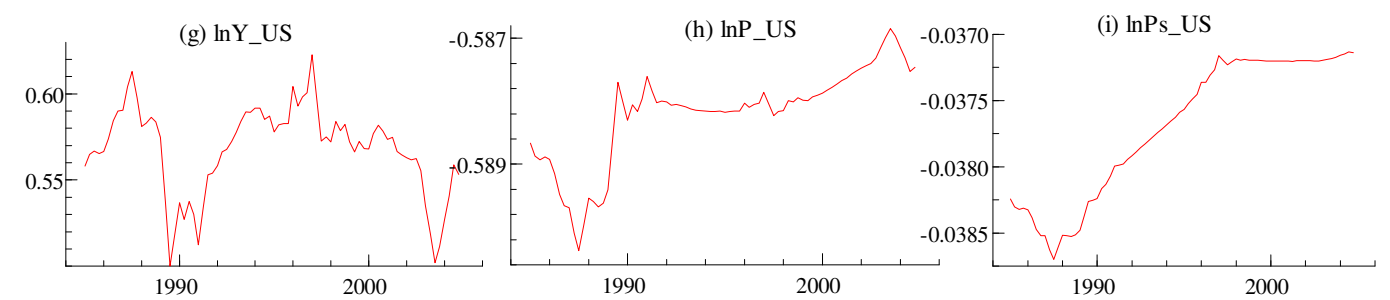

Figure 2. Estimation results of the TVP-STSMs at period 2004 4.

In general, the TVP-STSMs tend to have fewer significant explanatory variables than more conventional regression models such as autoregressive distributed lag models (ADLMs). This is because the unobserved components in a well-specified TVP-STSM are able to capture a large amount of the variation in the demand variable under study, and therefore the effects of some explanatory variables may no longer be statistically significant. However, they are retained in the final models if they are correctly signed, for the following two reasons. Firstly, these explanatory variables have commonly been identified as the most important determinants of the demand for Hong Kong tourism in past tourism demand studies (see, for example, Song, Wong, \& Chon, 2003). Secondly, including these variables enables the models to pass the diagnostic checks and to achieve strong convergence in model estimation. These estimated models are subsequently examined with regard to their forecasting performances relative to those of other commonly used econometric and time series methods.

The summary statistics in Table 1 indicate that the models are correctly specified, as all of the models reported pass all diagnostic tests at the 5\% significance level. In particular, no serial correlation exists in the residuals, which suggests that the models have captured the dynamic nature of the dependent variable adequately. Although the relative goodnessof-fit measure $R_{s}^{2}$ appears low in the Korea model, there is strong evidence that the TVPSTSM specification is preferable to a random walk plus drift and fixed seasonals model, 
which is the benchmark model in the $R_{s}^{2}$ calculation. For example, the TVP-STSM passes all diagnostic tests, the random walk estimates of the income and relative-price variables are both significant, and stochastic seasonality is evident, as can be seen from the plot in Figure 1(b).

With respect to the estimates of various hyperparameters, i.e., the average percentage deviations of the disturbances in the measurement and state equations, it can be seen that the tourist arrivals from different source markets exhibit different trends and seasonal patterns (see Table 1). All of the Hong Kong tourist arrivals series under study exhibit stochastic seasonality. For example, panels (b)-(d) of Figure 1 show clear evidence of varying seasonal patterns in the Korea, UK and US markets, particularly since the mid1990s, whereas panel (a) of Figure 1 shows that the seasonal pattern in the China market changes in a much more gradual fashion. Cycles do not feature in any markets over the observed sample period. This is consistent with most of the tourism demand literature using CSMs, such as the studies of González and Moral (1995) and Kulendran and Witt (2003). It indicates that the key economic determinants included have successfully explained the variation in the tourism demand variable, and therefore it is not necessary to include an additional cyclical component (Moosa, 2000). The initial trend specifications hold in two of the final models, those for Korea and the UK. This indicates that a smooth local linear trend specification fits the tourist arrivals series from these two countries well. For China and the US, deterministic slopes are sufficient to fit the arrivals series. With regard to the explanatory variables, the variances of the estimated coefficients make the time-varying nature of the various demand elasticities clear (see Table 1 and Figure 2). The income and own-price elasticities vary more significantly over time than the cross-price elasticities. In particular, the time-varying income elasticities are evident in all four markets under study. By contrast, a non-constant cross-price elasticity appears in the US market only.

With regard to the regression effects in the final state, the origin income shows the most significant effect (see Table 1). Although they are not always statistically significant in the final state, the estimated coefficients of the income and relative price variables have the expected signs in all cases, in line with demand theory.

As Table 1 shows, the TVP estimates of the income variable are significant at the 5\% significance level in both the China and Korea models, but insignificant in the UK and US markets. ${ }^{2}$ It seems that the effect of income on tourism demand is more evident in developing countries than in developed countries. With respect to the magnitudes of the estimated income elasticities, they vary across markets. As far as the two significant cases are concerned, the income elasticity is lower in the China market than in the Korea market. This is probably because of the high proportion of day-trips among the Chinese

\footnotetext{
${ }^{2}$ To provide robust findings, those results which are both economically and statistically significant are selected for discussion. When referring to the quantitative nature of the point estimates, statistically insignificant results are omitted.
} 
visitors who travel to Hong Kong for business purposes. On the contrary, most Korean visitors are overnight leisure tourists, and therefore their income elasticity is expected to be higher. Panels (a) and (c) of Figure 2 illustrate the time-varying effects of income on tourism demand from the China and Korea markets, respectively. Both graphs show a turning point in the late 1990s, but opposite trends were followed afterwards. The decreasing income elasticity in the Korea market suggests the maturity of this market as far as the destination of Hong Kong is concerned. On the other hand, as an emerging market, China has experienced a boom in outbound travel in recent years that is reflected in the growth in tourism to Hong Kong. This is mainly attributable to the strong, stable economic growth in China. With respect to the own-price elasticities, the results generally suggest that the demand for Hong Kong tourism has become less sensitive to the variations in tourism prices in Hong Kong relative to those in the origin countries since the early 1990s. This is particularly evident in the Korea market, as panel (d) of Figure 2 shows.

Substitute prices seem to be important for the UK market, but less important for the other origins. In particular, the significant positive coefficient in the case of the UK suggests that destinations neighbouring Hong Kong are strongly regarded as substitutes by UK tourists.

As far as the intervention effects of the major one-off events (which are estimated as fixed parameters) are concerned, the SARS outbreak in 2003 had the most significant impact on tourist arrivals from all of the source markets. It should be noted that although the war in Iraq also occurred in 2003, it did not have a significant impact on tourist arrivals to Hong Kong. The statistics for UK and US outbound tourism both show a positive growth in 2003, indicating that the war in Iraq did not have a significant effect on the outbound travel from these two countries. Therefore, the dummy variable for 2003 only captures the effect of the SARS outbreak. The handover of Hong Kong to China in 1997 is observed to have had a significant adverse effect on the UK market, but the effect on the mainland Chinese market is insignificant. The Asian financial crisis in 1997 did not have a significant impact on Korean tourist arrivals to Hong Kong. The September 2001 terrorist attack did not have any significant impact on tourism demand in Hong Kong either, except for the US market. The effect of the 1990-1991 Gulf war is not significant in any of the models. Hence, it is removed from the final model estimation.

\subsection{Forecast accuracy analysis}

To assess the forecast accuracy of the TVP-STSMs in comparison with that of other econometric and time series models, one-, two-, three-, four- and eight-quarters-ahead forecast horizons are considered, and ex-post dynamic forecasts are generated over the hold-out period 2005Q1-2008Q4. A recursive forecasting technique is used during the whole procedure, i.e., the models are first estimated over the period 1985Q1 to 2004Q4, then this estimated model is used to forecast Hong Kong inbound tourist arrivals from each of the four origin countries. The models are then re-estimated for the periods up to 2005Q1, 2005Q2,.., 2008Q3, and forecasts are generated based on each re-estimated model for 2005Q2-2008Q4, 2005Q3-2008Q4,.., 2008Q4, respectively. As a result, 16 
one-step-ahead forecasts, 15 two-steps-ahead forecasts, 14 three-steps-ahead forecasts, 13 four-steps-ahead forecasts and 9 eight-steps-ahead forecasts are generated for each source market under study. The forecast accuracy is evaluated based on the mean absolute percentage error (MAPE) and the root mean square percentage error (RMSPE). The seasonal naïve "No Change 1" (constant value for each season) and "No Change 2" (constant growth rate for each season) models, the SARIMA model, BSM, CSM, the TVP model, and ADLM are included in the comparison of the forecasting performances. Due to space constraints, the specifications of these models are omitted here, but technical details are given by Frechtling (2001) for the seasonal naïve models and Kulendran and Witt (2003) and Song and Witt (2000) for the rest.

\subsubsection{Ex-post forecast accuracy comparison rankings}

The results for the ex-post forecast accuracy comparisons are reported in Table 2. Over all of the forecasting horizons being considered, the TVP-STSM is consistently ranked first among the eight competing models, as judged by both MAPE and RMSPE with only one exception. For the shorter term (one to three quarters ahead) the TVP-STSM, CSM, TVP model and BSM, all of which belong to the family of state space models, take the top four positions in the forecasting competition according to the MAPE. The very good short-term forecasting performances of the TVP model, CSM and BSM are in line with the findings of past tourism forecasting studies, such as those of Song and Witt (2000), González and Moral (1995) and Turner and Witt (2001). In particular, the TVP-STSM, CSM and TVP model, all of which incorporate explanatory variables into the model specifications, generate the most accurate one- and two-quarters-ahead forecasts. This indicates that introducing explanatory variables may help improve the short-term forecast accuracy of state space models. The outstanding forecasting performances of the TVPSTSM, CSM and BSM suggest that it is most appropriate to model seasonal tourism demand using the unobserved components method, which incorporates stochastic specifications of trend, seasonal and cycle components. Such specifications help to capture the dynamics of seasonal tourism demand as accurately as possible. The implication of the superior performance of the TVP-STSM compared to CSM is that the demand features that are not explained by the unobserved components are likely to embody stochastic patterns, which are better modelled as time-varying parameters of the explanatory variables. Looking at the relative forecast accuracies of the TVP-STSM and TVP model, which use the same estimation method for the explanatory variables (i.e., the Kalman filter algorithm), the results show that applying the TVP model to seasonal tourism demand forecasting simply by augmenting the original TVP specification with deterministic seasonal dummies is not sufficient to capture the dynamic patterns of seasonal tourist arrivals. 
Table 2. Comparison of ex post forecasting accuracy rankings over different forecasting horizons.

\begin{tabular}{|c|c|c|c|c|c|c|c|c|c|}
\hline Horizon & Measure & TVP-STSM & CSM & TVP & BSM & ADLM & SARIMA & Naïve 1 & Naïve 2 \\
\hline \multirow{2}{*}{1 quarter } & MAPE & $0.437(1)$ & $0.528(2)$ & 0.615 (3) & $0.625(4)$ & $0.849(6)$ & $0.744(5)$ & $0.895(7)$ & $1.793(8)$ \\
\hline & RMSPE & $0.590(1)$ & $0.721(2)$ & $0.876(4)$ & $0.882(5)$ & $0.903(6)$ & $0.820(3)$ & $1.000(7)$ & $2.625(8)$ \\
\hline \multirow[t]{2}{*}{2 quarters } & MAPE & $0.554(1)$ & $0.642(2)$ & 0.680 & $0.705(4)$ & $0.937(7)$ & $0.910(6)$ & $0.872(5)$ & $1.804(8)$ \\
\hline & RMSPE & $0.720(1)$ & 0.818 & 0.957 (3) & $1.018(7)$ & $1.017(6)$ & $1.000(5)$ & $0.973(4)$ & $2.680(8)$ \\
\hline \multirow[t]{2}{*}{3 quarters } & MAPE & 0.637 (1) & $0.771(3)$ & $0.852(4)$ & $0.747(2)$ & $1.118(8)$ & $0.958(6)$ & $0.856(5)$ & $1.048(7)$ \\
\hline & RMSPE & $0.860(1)$ & $0.983(3)$ & $1.126(6)$ & $0.985(4)$ & $1.225(8)$ & $1.045(5)$ & $0.953(2)$ & 1.147 (7) \\
\hline \multirow[t]{2}{*}{4 quarters } & MAPE & $0.733(1)$ & $0.912(5)$ & $0.851(3)$ & $0.802(2)$ & $1.280(8)$ & $0.956(6)$ & 0.869 (4) & $0.970(7)$ \\
\hline & RMSPE & $0.910(1)$ & $1.124(7)$ & $1.121(6)$ & $1.015(3)$ & $1.409(8)$ & 1.029 (4) & $0.963(2)$ & $1.069(5)$ \\
\hline \multirow[t]{2}{*}{8 quarters } & MAPE & $1.053(1)$ & $1.660(7)$ & $1.330(5)$ & $1.124(2)$ & $1.780(8)$ & $1.250(3)$ & $1.469(6)$ & $1.308(4)$ \\
\hline & RMSPE & $1.309(2)$ & $2.050(8)$ & $1.716(6)$ & $1.302(1)$ & $1.997(7)$ & $1.365(3)$ & $1.616(5)$ & $1.452(4)$ \\
\hline
\end{tabular}

Note: The figures in parentheses denote rankings. 
As the forecasting horizon is extended, the TVP model and CSM generate relatively less accurate forecasts. On the other hand, two time series models, the BSM and the SARIMA model, show improved forecasting results. In particular, the BSM outperforms all of its competitors except the TVP-STSM over the eight-quartersahead horizon according to MAPE. This indicates that for an econometric model to outperform its time series counterparts in the longer-term (four and eight quarters ahead) forecasting of seasonal tourism demand, both stochastic seasonality and TVP estimation of explanatory variable coefficients should be considered.

The ADLM that incorporates deterministic seasonal dummies in the model specification always shows poor forecasting performance in the group. Similarly, the two seasonal naïve models that also assume deterministic seasonality fail to generate satisfactory forecasts over most horizons. Once again, this comparison implies that treating seasonality as stochastic in a forecasting model's specification is more appropriate than regarding seasonality as deterministic.

\subsubsection{HLN statistical test of differences in forecasting accuracy}

The Harvey-Leybourne-Newbold (HLN) test proposed by Harvey, Leybourne, and Newbold (1997) is applied to examine statistically significant differences in forecasting accuracy between the TVP-STSM and the competing models. As Table 3 shows, the TVP-STSM clearly outperforms all competing models at the 5\% significance level for one-quarter-ahead forecasts, and at the $10 \%$ significance level for two-quarters-ahead forecasts. With respect to the three-quarters-ahead forecasts, statistical evidence can be found for the TVP-STSM's superior forecasting performance at the $10 \%$ significance level in all but one case (BSM). As the forecasting horizon extends, the forecast accuracy declines for all models. Although the TVP-STSM's superiority is less evident statistically speaking, its four- and eightquarters-ahead forecasts are still statistically more accurate than those of the CSM (at the $10 \%$ significance level) and ADLM (at the 1\% significance level).

Table 3. HLN statistical tests of the differences in forecasting accuracy levels between TVP-STSM and other models.

\begin{tabular}{llllllll}
\hline Horizon & CSM & TVP & BSM & ADLM & SARIMA & Naïve 1 & Naïve 2 \\
\hline 1 quarter & $3.244^{* * *}$ & $3.400^{* * *}$ & $1.923^{* *}$ & $5.575^{* * *}$ & $3.225^{* * *}$ & $4.141^{* * * *}$ & $3.247^{* * *}$ \\
2 quarters & $1.386^{*}$ & $1.761^{* *}$ & $1.308^{*}$ & $3.717^{* * *}$ & $2.858^{* * *}$ & $2.818^{* * *}$ & $2.766^{* * *}$ \\
3 quarters & $1.438^{*}$ & $2.472^{* * *}$ & 1.243 & $3.007^{* * *}$ & $2.326^{* *}$ & $1.589^{*}$ & $2.932^{* * *}$ \\
4 quarters & $1.403^{*}$ & 0.937 & 0.573 & $3.051^{* * *}$ & 1.210 & 0.852 & $1.572^{*}$ \\
8 quarters & $1.339^{*}$ & 0.745 & 0.227 & $2.217^{* *}$ & 0.571 & 1.005 & 0.687 \\
\hline
\end{tabular}

Note: $*$, $* *$ and $* * *$ denote significance at $10 \%, 5 \%$ and $1 \%$ levels (one-tailed tests).

\subsubsection{Ex-ante forecast accuracy comparison rankings}

As has been previously discussed in Section 2, models with explanatory variables have the great advantage over univariate time series models of incorporating the impact of important economic determinants on tourism demand. However, the greatest disadvantage of these models is that when forecasting into the future (where no variables have yet been observed) one must produce forecasts of the regressors before forecasting the regressand. In many cases one could argue that it may actually 
be more challenging to forecast the regressors than to forecast the dependent variable directly. In this section we examine the effect of not observing the explanatory variables over the hold-out sample, but forecasting them. Hence, all forecasting is now performed ex-ante. In order to forecast the explanatory variables, exponential smoothing methods are employed by implementing the fully automated algorithm for forecasting univariate time series using innovations state space models, as presented by Hyndman and Khandakar (2008) (see also Hyndman, Koehler, Ord, \& Snyder, 2008). The ex-ante forecasting evaluation results are presented in Table 4.

As expected, both the MAPEs and RMSPEs have deteriorated (increased) for most models with explanatory variables when performing ex-ante forecasting compared to ex-post. The only exception is for the TVP models, where both forecast error measures improve (i.e., become smaller) for all forecast horizons. This somewhat counterintuitive and surprising result has also appeared in other studies (see for example the discussion by Athanasopoulos, Hyndman, Song, \& Wu, 2010, and references therein, Section 5.5).

When comparing the rankings of the models between ex-post and ex-ante forecasting, it is found that the TVP-STSM is still ranked first among all methods for forecasting one quarter ahead. For longer horizons, the TVP model and the BSM mostly hold the number one and number two rankings between them, with the TVP-STSM being mostly ranked third. Although the performance of the TVP-STSM has deteriorated compared to ex-post forecasting, it still produces satisfactory forecasts. This is reflected in the results of the HLN tests, presented in Table 5, where the TVP-STSM shows significantly more accurate one-quarter-ahead forecasts over all its competitors except the TVP model, and no methods predict significantly better than the TVPSTSM for longer horizons. 
Table 4. Comparison of ex ante forecasting accuracy rankings over different forecasting horizons.

\begin{tabular}{|c|c|c|c|c|c|c|c|c|c|}
\hline Horizon & Measure & TVP-STSM & CSM & TVP & BSM & ADLM & SARIMA & Naïve 1 & Naïve 2 \\
\hline \multirow[t]{2}{*}{1 quarter } & MAPE & 0.531 (1) [1] & 0.580 (3) [2] & $0.573(2)[3]$ & 0.625 (4) [4] & $0.882(6)[6]$ & $0.744(5)[5]$ & $0.895(7)[7]$ & $1.793(8)[8]$ \\
\hline & RMSPE & 0.736 (1) [1] & 0.826 (4) [2] & $0.786(2)[4]$ & $0.882(5)[5]$ & $1.150(7)[6]$ & 0.820 (3) [3] & $1.000(6)[7]$ & $2.625(8)[8]$ \\
\hline \multirow[t]{2}{*}{2 quarters } & MAPE & $0.738(3)[1]$ & 0.748 (4) [2] & 0.665 (1) [3] & 0.705 (2) [4] & 0.991 (7) [7] & $0.910(6)[6]$ & $0.872(5)[5]$ & $1.804(8)[8]$ \\
\hline & RMSPE & $0.935(2)[1]$ & $1.006(5)[2]$ & 0.922 (1) [3] & $1.018(6)[7]$ & $1.324(7)[6]$ & 1.000 (4) [5] & 0.973 (3) [4] & $2.680(8)[8]$ \\
\hline \multirow[t]{2}{*}{3 quarters } & MAPE & $0.812(2)[1]$ & 0.907 (5) [3] & 0.841 (3) [4] & 0.747 (1) [2] & $1.123(8)[8]$ & $0.958(6)[6]$ & 0.856 (4) [5] & $1.048(7)[7]$ \\
\hline & RMSPE & 1.053 (4) [1] & 1.188 (7) [3] & $1.071(5)[6]$ & 0.985 (2) [4] & $1.474(8)[8]$ & 1.045 (3) [5] & 0.953 (1) [2] & $1.147(6)[7]$ \\
\hline \multirow[t]{2}{*}{4 quarters } & MAPE & 0.809 (3) [1] & $0.966(6)[5]$ & 0.768 (1) [3] & $0.802(2)[2]$ & $1.211(8)[8]$ & $0.956(5)[6]$ & 0.869 (4) [4] & 0.970 (7) [7] \\
\hline & RMSPE & 1.015 (3) [1] & 1.234 (7) [7] & 0.998 (2) [6] & 1.015 (3) [3] & $1.554(8)[8]$ & $1.029(5)[4]$ & 0.963 (1) [2] & $1.069(6)[5]$ \\
\hline \multirow[t]{2}{*}{8 quarters } & MAPE & $1.244(3)[1]$ & $1.358(6)[7]$ & $1.197(2)[5]$ & $1.124(1)$ [2] & $1.420(7)[8]$ & 1.250 (4) [3] & 1.469 (8) [6] & $1.308(5)[4]$ \\
\hline & RMSPE & $1.505(5)[2]$ & $1.819(8)[8]$ & $1.402(3)[6]$ & $1.302(1)[1]$ & $1.794(7)[7]$ & $1.365(2)[3]$ & $1.616(6)[5]$ & $1.452(4)[4]$ \\
\hline
\end{tabular}

Note: The figures in parentheses denote rankings. The figures in the square brackets denote rankings when ex-post forecasting was performed; these are extracted from Table 2. 
Table 5. HLN statistical tests of differences in ex ante forecasting accuracy levels between TVP-STSM and other models.

\begin{tabular}{llllllll}
\hline Horizon & CSM & TVP & BSM & ADLM & SARIMA & Naïve 1 & Naïve 2 \\
\hline 1 quarter & $2.435^{* * *}$ & 0.577 & $2.467 * * *$ & $4.015^{* * * *}$ & $3.068^{* * *}$ & $4.078^{* * * *}$ & $2.999^{* * *}$ \\
2 quarters & 0.211 & -1.295 & -0.242 & $2.106^{* * *}$ & $1.310^{*}$ & $1.687^{* *}$ & $2.359^{* *}$ \\
3 quarters & 1.024 & 0.348 & -0.654 & $2.738^{* * *}$ & 1.128 & 0.566 & $1.673^{*}$ \\
4 quarters & 1.071 & -0.459 & -0.073 & $2.944^{* * *}$ & 1.209 & 0.858 & 1.045 \\
8 quarters & 0.241 & -0.252 & -0.501 & 0.768 & 0.023 & 1.241 & 0.148 \\
\hline
\end{tabular}

Note: $* * *$ and $* * *$ denote significance at $10 \%, 5 \%$ and $1 \%$ levels (one-tailed tests).

\section{Concluding remarks}

This study has developed a complete stochastic parameter model, the TVP-STSM, in which all of the parameters of the trend, seasonal, cycle and explanatory variables are treated as time-varying. This TVP-STSM has been used to model tourist arrivals to Hong Kong from four key source markets. In addition, the forecasting performance of the TVP-STSM has been examined and compared with that of seven commonly used time series and econometric forecasting models.

The empirical results show that the newly developed TVP-STSM consistently outperforms time series models, such as the Naïve 1 and Naïve 2 models, the SARIMA model and BSM, and econometric forecasting models, such as the TVP model, CSM and ADLM, for one- to four- and eight-quarter-ahead forecasting. Its outstanding performance is particularly evident for one- to three-quarters-ahead expost forecasting, and one-quarter-ahead ex-ante forecasting. Other state space models, including the CSM, BSM and the TVP model, all generate sound short-term (one to three quarters ahead) forecasts. These results suggest that it is important to treat seasonality as stochastic (BSM and CSM), and also to estimate explanatory variables as time-varying (TVP model) when modelling and forecasting seasonal tourism demand, and that it is most beneficial to consider both jointly in one forecasting model (TVP-STSM).

Although this study has demonstrated the advantages of the TVP-STSM over various time series and econometric models, more research still needs to be carried out. Firstly, with longer datasets, the significance of the TVP estimates of the explanatory variables in a TVP-STSM is likely to be improved. The estimation of such a complex model with large numbers of parameters and hyperparameters consumes a considerable number of degrees of freedom and is likely to affect the significance of the estimates. Secondly, the TVP-STSM should be tested on a variety of origindestination pairs, in order to provide more robust empirical evidence on the merits of this new forecasting method. Thirdly, more econometric and time series models could be introduced into the comparison in order to test the forecasting ability of the TVPSTSM further. 


\section{Acknowledgements}

The authors would like to acknowledge the financial support of the Hong Kong UGCGRF grant PolyU 5473/06H and data support from Wei Guo. Athanasopoulos wishes to acknowledge the financial support from the Australian Research Council grant DP0984399.

\section{Bios}

Haiyan Song is Chair Professor of Tourism in the School of Hotel and Tourism Management at The Hong Kong Polytechnic University. His main research interest is in tourism demand modeling and forecasting with a particular focus on the evaluation of forecasting performance of various econometric models in the context of tourism.

Gang Li is Senior Lecturer in Economics in the Faculty of Management and Law at the University of Surrey. Dr Li's research interests include tourism economics and tourism demand forecasting.

Stephen F. Witt is Emeritus Professor of Tourism Forecasting in the Faculty of Management and Law at the University of Surrey. His main research area is in tourism demand forecasting.

George Athanasopoulos is Senior Lecturer in the Department of Econometrics and Business Statistics, Monash University, and his research interests are in the areas of time series analysis and forecasting.

\section{References}

Athanasopoulos, G., Hyndman, R. J., Song, H., \& Wu, D. (2010). The tourism forecasting competition. International Journal of Forecasting, forthcoming.

Butler, R. (1994). Seasonality in tourism: Issues and problems. In A. Seaton (Ed.), Tourism: The state of the art (pp.332-339). Chichester: Wiley.

Commandeur, J. J. F., \& Koopman, S. J. (2007). An introduction to state space time series analysis. New York: Oxford University Press.

Durbin, J., \& Koopman, S. J. (2001). Time series analysis by state space methods. New York: Oxford University Press.

Frechtling, D. C. (2001). Forecasting tourism demand: Methods and strategies. Oxford: Butterworth-Heinemann.

González, P., \& Moral, P. (1995). An analysis of the international tourism demand in Spain. International Journal of Forecasting, 11, 233-251.

González, P., \& Moral, P. (1996). Analysis of tourism trend in Spain. Annals of Tourism Research, 23, 739-754.

Hamilton, J. D. (1994a). Time series analysis (Chapter 13). Princeton, NJ: Princeton University Press.

Hamilton, J. D. (1994b). State space models. In R.F. Engle \& D.L. McFadden (Eds.), Handbook of econometrics (Vol. 4, pp. 3041-3080). Amsterdam: Elsevier Science B.V.

Harrison, P. J., \& Stevens, C. F. (1976). Bayesian forecasting (with discussion). Journal of Royal Statistical Society, Series B, 38, 205-247. 
Harvey, A. C. (1989). Forecasting structural time series models and the Kalman filter. New York: John Wiley.

Harvey, D., Leybourne, S., \& Newbold, P. (1997). Testing the equality of prediction mean squared errors. International Journal of Forecasting, 13, 281-291.

Hyndman, R. J., \& Khandakar, Y. (2008). Automatic time series forecasting: The forecast package for R, Journal of Statistical Software, 26, 1-22.

Hyndman, R. J., Koehler, A. B., Ord, K. \& Snyder, R. D., (2008). Forecasting with exponential smoothing: the state space appraoch. Springer-Verlag: BerlinHeidelberg.

Koopman, S. J., Harvey, A. C., Doornik, J. A., \& Shephard, N. (2007). Structural time series analyser, modeller and predictor: STAMP 8. London: Timberlake Consultants.

Koopman, S. J., Shephard, N., \& Doornik, J. A. (1999). Statistical algorithms for models in state space using SSF pack 2.2. Econometrics Journal, 2, 107-160.

Kulendran, N., \& King, M. (1997). Forecasting international quarterly tourism flows using error correction and time series models. International Journal of Forecasting, 13, 319-327.

Kulendran, N., \& Witt, S. F. (2001). Cointegration versus least squares regression. Annals of Tourism Research, 28, 291-311.

Kulendran, N., \& Witt, S. F. (2003). Forecasting the demand for international business tourism. Journal of Travel Research, 43, 141-150.

Li, G., Song, H., \& Witt, S. F. (2005). Recent developments in econometric modeling and forecasting. Journal of Travel Research, 44, 82-99.

Lucas, J. R. E. (1976). Econometric policy evaluation: A critique. In K. Brumer \& A. H. Meltzer (Eds.), The Philip curve and labour markets (Vol. 1, pp. 19-46). Carnegie Rochester Series on Public Policy, Journal of Monetary Economics (suppl.).

Moosa, I. A. (2000). The cyclical behaviour of prices in the U.K.: Some structural time series evidence. Empirical Economics, 25, 261-278.

Shen, S., Li, G., \& Song, H. (2008). An assessment of combining tourism demand forecasts over different time horizons. Journal of Travel Research, 47, 197-207.

Smeral, E., Witt, S. F., \& Witt, C. A. (1992). Econometric forecasts: Tourism trends to 2000. Annals of Tourism Research, 19, 450-466.

Song, H., \& Witt, S. F. (2000). Tourism demand modelling and forecasting: Modern econometric approaches. Oxford: Pergamon.

Song, H., Witt, S. F., \& Jensen, T. C. (2003). Tourism forecasting: Accuracy of alternative econometric models. International Journal of Forecasting, 19, 123141.

Song, H., Wong, K. F., \& Chon, K. S. (2003). Modelling and forecasting demand for Hong Kong tourism. International Journal of Hospitality Management, 22, 435451.

Turner, L. W., \& Witt, S. F. (2001). Forecasting tourism using univariate and multivariate structural time series models. Tourism Economics, 7, 135-147. 
Witt, S. F., \& Witt, C. A. (1995). Forecasting tourism demand: A review of empirical research. International Journal of Forecasting, 11, 447-475.

Witt, S. F., Song, H., \& Louvieris, P. (2003). Statistical testing in forecasting model selection. Journal of Travel Research, 42, 151-158. 